University of Nebraska - Lincoln

DigitalCommons@University of Nebraska - Lincoln

Faculty Publications, Classics and Religious

Studies Department

2009

\title{
Jüdische Vermittler des Hebräischen und ihre christlichen Schüler im Spätmittelalter
}

Stephen G. Burnett

University of Nebraska - Lincoln, sburnett1@unl.edu

Follow this and additional works at: https://digitalcommons.unl.edu/classicsfacpub

Part of the Classics Commons

Burnett, Stephen G., "Jüdische Vermittler des Hebräischen und ihre christlichen Schüler im Spätmittelalter" (2009). Faculty Publications, Classics and Religious Studies Department. 110. https://digitalcommons.unl.edu/classicsfacpub/110

This Article is brought to you for free and open access by the Classics and Religious Studies at DigitalCommons@University of Nebraska - Lincoln. It has been accepted for inclusion in Faculty Publications, Classics and Religious Studies Department by an authorized administrator of DigitalCommons@University of Nebraska - Lincoln. 


\title{
Wechselseitige Wahrnehmung der Religionen im Spätmittelalter und in der Frühen Neuzeit
}

\author{
I. Konzeptionelle Grundfragen \\ und Fallstudien (Heiden, Barbaren, Juden)
}

Herausgegeben von

Ludger Grenzmann, Thomas Haye, Nikolaus Henkel und Thomas Kaufmann

Walter de Gruyter · Berlin · New York 


\title{
Jüdische Vermittler des Hebräischen und ihre christlichen Schüler im Spätmittelalter
}

\author{
STEPHEN G. BurnetT
}

Bruder Konrad Pellikan, ein Franziskanermönch, war das heroische Beispiel eines christlichen Autodidakten der hebräischen Sprache. Im Jahre 1499 fing er sein Studium des Hebräischen auf der Basis seiner Analyse des hebräischen Bibeltextes mit einer wortwörtlichen lateinischen Übersetzung der späteren Prophetenbücher ${ }^{1}$ und auch mit den transkribierten Versen von Jesajas, die sich in Petrus Nigris Stella Messiae befanden, an. Im Juli des folgenden Jahres hatte er bei einem Besuch in Tübingen von Reuchlin eine Erklärung der Nennform (Infinitiv) des Verbs bekommen. Im August besuchte er den Ulmer Priester Johannes Böhm. Dieser stellte ihm zwei handschriftliche Fragmente von Moses Kimhis Grammatik zur Verfügung, damit Pellikan sie kopieren konnte. ${ }^{2}$ Als er im Dienst des Franziskaner Ordens reiste, besuchte er andere Gelehrte und Bibliotheken, um weitere Hebräischkenntnisse zu gewinnen und auch um hebräische Handschriften zu lesen und zu kopieren. Seine grammatikalische Skizze der hebräischen Sprache ist schon 1504, drei Jahre vor Reuchlins Rudimenta, im Druck erschienen. ${ }^{3}$ Trotz all seiner Anstrengungen brauchte er schliesslich doch jüdische Hilfe, um seine Kenntnisse zu verbessern. Im Jahre 1513 besuchte er den Unterricht bei Matthäus Adrianus, einem getauften Juden aus Spanien. ${ }^{4}$ Auch Pellikan, der Autodidakt des Spätmittelalters, musste jüdische Hilfe in Anspruch nehmen, um Fortschritte zu erzielen.

1 Die Handschrift war ein Geschenk von Paulus von Pfedersheim, einem getauften Juden und Franziskanermönch. Konrad Pellikan: Die Hauschronik Konrad Pellikans von Rufach. Ein Lebensbild aus der Reformationszeit. Übers. ThEOdOR Vulpinus. Straßburg 1892, S. 18. Nach der Germania Judaica (Bd. 3: 1350-1519, 2. Teilband: Ortschaftenartikel Märisch-Budwitz-Zwolle. Hg. von Arye Maimon s. A., Mordechai Breuer und Yacov Guggenheim. Tübingen 1995, S. 1105, A. 20) ist Paulus von Pfedersheim wahrscheinlich nicht mit Johannes Pauli identisch. - Ich möchte meiner Kollegin Frau Professor Dr. Susanna Schrafstetter (University of Nebraska-Lincoln) und Herrn Dr. Grenzmann für ihre Hilfe bei der Korrektur des Aufsatzes danken.

2 Thомаs Willi: Der Beitrag des Hebräischen zum Werden der Reformation in Basel. Theologische Zeitschrift 35 (1979) S. 139-154, hier S.141. Vgl. auch Bernhard Walde: Christliche Hebraisten Deutschlands am Ausgang des Mittelalters. Münster i. W. 1916, S. 190-94.

3 Willi: Der Beitrag des Hebräischen (wie A. 2), S. 142.

4 Ebd. S. 144. 
Wenn selbst Pellikan jüdische Hilfe brauchte, war das bestimmt umso nötiger für andere christliche Schüler der hebräischen Sprache, die weniger sprachbegabt waren als er, und die mehr Geld zu Verfügung hatten und bereit waren, die oft hohen Preise für einen Unterricht zu bezahlen. Jüdische Vermittler, ob Juden oder getaufte Juden, spielten eine Schlüsselrolle bei der Gründung der Hebraistik als eines akademischen Faches, vor allem in Italien und Deutschland während des Spätmittelalters und der Frühreformationszeit. ${ }^{5}$ Es gab keine große Zahl dieser Vermittler (ich nenne etwas mehr als 30 Namen - es gab einige mehr), aber ihre Rolle als erfahrene Experten oder mindestens als ,Insider' der Jüdischen Welt war bedeutend. Aufgrund ihrer Kenntnis der jüdischen Sprache und Texte, ihrer Fähigkeiten als Schreiber und Übersetzer solcher Texte und - mindestens ebenso - aufgrund ihrer Fähigkeit, die Grenzen der zwei Welten, der jüdischen und der christlichen, zu überschreiten, sind diese jüdischen Vermittler Katalysatoren für das Wachstum der Hebraistik, vor allem in Italien und Deutschland.

Einige dieser jüdischen Vermittler sind in der Wissenschaft schon längst bekannt. Aber meine Analyse zielt nicht in erster Linie auf Persönlichkeiten, sondern auf das Profil dieser Vermittler als einer Gruppe, auf die sozialen Kontexte ihrer Verdienste in Italien und Deutschland und auf ihre Dienstleistungen als Katalysatoren der christlichen Hebraistik in diesen zwei Regionen.

\section{Profil der jüdischen Vermittler}

Die jüdischen Helfer, die die Hebraistik unter Christen möglich machten, waren natürlich nicht alle gleich, wenn man sie nach Persönlichkeit, Gelehrsamkeit oder ihrer Position innerhalb oder ausserhalb der jüdischen Gesellschaft betrachtet. Trotzdem sind einige Gemeinsamkeiten zwischen ihnen bemerkenswert. Erstens sind einige der bedeutendsten Lehrer Ärtzte mit Universitätsausbildung, Juden und getaufte Juden gleichermaßen. David Ruderman hat in seiner Forschung über die Zulassung jüdischer Medizinstudenten von der Promovierung jüdischer Ärtzte an der Universität Padua ab 1517 und von ihrer Bedeutung als Vermittler neuer Perspektiven über Medizin und der Naturwissenschaften unter Juden in der frühen Neuzeit berichtet. Er betonte auch, dass das Studium in Padua diesen jüdischen Studenten eine allgemeine Ausbildung in den philosophischen Fächern anbot. ${ }^{6}$ Diese Studenten waren deshalb fähiger

5 Jerome Friedman: The Most Ancient Testimony: Christian Hebraica in the Age of Renaissance Nostalgia. Athens, OH, 1983, S. 38-49.

6 David B. Ruderman: The Impact of Science on Jewish Culture and Society in Venice. In: Essential Papers on Jewish Culture in Renaissance and Baroque Italy. Hg. von DAviD 
als die Mehrheit der Juden, Kontakte mit ausgebildeten Christen innerhalb und außerhalb der Akademie anzuknüpfen.

Abraham de Balmes, Obadja Sforno und Jacob Martino haben alle ein Universitätsstudium in Medizin absolviert - wie auch die getauften Juden Matthaeus Adrianus, Paulus Ricius und Paul Weidner. Ein Universtätsstudium war nicht unbedingt nötig, um die Befähigung zum Hebräischlehrer zu erlangen. Sonst hätte Elias Levita keine Karriere machen können; er war ohne Zweifel der beste Hebräischlehrer seiner Zeit. Trotzdem erleichterte ein Studium den Umgang mit Christen gleicher Ausbildung.

Dass diese Hebräischlehrer Mediziner waren, ist auch deshalb wichtig, weil sie wegen ihres Berufs von Christen leichter als andere Juden zu besuchen und zu konsultieren waren. Die italienischen Ärzte waren zudem oft prominente Figuren. Obadjah Sforno war Leibarzt von Papst Alexander VI., als er Reuchlin unterrichtete. De Balmes war zugleich Leibarzt von Kardinal Grimani und auch außerordentlicher Professor für Medizin an der Universität Padua. Jacob Jehiel Loans aus Mantua, Reuchlins wichtigster jüdischer Lehrer in Deutschland, war Leibarzt von Kaiser Friedrich III. ${ }^{7}$ Obwohl diese Hebräischlehrer Juden waren, konnte man ohne weiteres ihre Hilfe anerkennen. Hinsichtlich der Hilfe von Juden, deren Ruf für Gelehrsamkeit nur innerhalb der jüdischen Welt bekannt war, musste man viel vorsichtiger sein, wenigstens in Deutschland. Ob und von wem die Übersetzer der Wormser Propheten Ludwig Hätzer und Hans Denck jüdische Hilfe bekommen haben, bleibt eine offene Frage. ${ }^{8}$ Sebastian Münster war überaus vorsichtig, wenn er überhaupt seinen jüdischen Helfern (außer Elias Levita, der in Italien wohnte und mit dem er wahrscheinlich keinen persönlichen Kontakt hatte) Anerkennung zollte. ${ }^{9}$ Normalerweise schrieb er: Ich habe von einem gelehrten Juden gehört. ${ }^{10}$

Eine gute Kenntnis der lateinischen Sprache war auch ein gemeinsamer Faktor bei vielen dieser jüdischen Hebräischlehrer. Sie war natürlich nötig für

B. Ruderman. New York 1992, S. 519-553, hier S. 521-24, und inem: Jewish Thought and Scientific Discovery in Early Modern Europe. New Haven, 1995, S. 100-17.

7 Saverio Campanini: Reuchlins Jüdische Lehrer aus Italien. In: Reuchlin und Italien. Hg. von Gerald Dörner. Stuttgart 1999 (Pforzheimer Reuchlinschriften, Band 7), S. 69-85, hier S. 76. - Abkürzungen im Folgenden: RBW = Johannes Reuchlin Briefwechsel. Hg. von Stefan Rhein, Matthias Dall'Asta und Gerald Dörner. Stuttgart-Bad Cannstatt, 1999. - RBW. Leseausgabe = Briefwechsel. Übers. AdalberT WeH. Stuttgart-Bad Cannstatt 2000.

8 James Beck: The Anabaptists and the Jews: the Case of Hätzer, Denck and the Worms Prophets. Mennonite Quarterly Review 75 (2001), S. 407-27, hier S. 415-23.

9 Gérard E. Weil: Élie Lévita. Humaniste et Massorète. Leiden 1963, S. 229-33.

$10, \ldots$ a docto quodam Judaeo, wie er denn überhaupt Umgang mit jüdischen Gelehrten gehabt zu haben scheint, ohne dass er aber je einen Namen nennt. “Zitiert von Joseph Prijs: Die Basler hebräischen Drucke. Erg. u. hg. von Bernhard Prijs. Olten 1964, S. 43. Vgl. Karl Heinz Burmeister: Sebastian Münster. Versuch eines biographischen Gesamtbildes. Basel und Stuttgart 1963, S. 72-76. 
diejenigen mit Universitätsverbindungen. ${ }^{11}$ Aber nicht alle jüdischen Lehrer waren gleich geschickt in der Sprache. Bernhard Göppingen hatte einen sehr schlechten Ruf als Lehrer, da er kein einziges Wort Latein oder Griechisch konnte. Eine solche Aussage war ziemlich unfair, da Bernhard etwas Latein konnte (Luthers Widmungsbrief an ihn in Dass Jesus Christus ein geborener Juden Sei [1523] war auf Lateinisch abgefasst), aber dennoch reichte sein Latein nicht aus, um seine provisorische Stelle an der Universität Wittenberg zu halten. ${ }^{12}$ Matthäus Adrianus hatte auch einen schlechten Ruf, nicht nur, weil er ein komplizierter Mensch war, sondern auch, weil er ein "regelrechtes vulgär Lateinisch“ gesprochen hat (so Alfred Hartmann), was nur mit großen Schwierigkeiten zu verstehen war. ${ }^{13}$ Michael Adam, Pellikans Hauslehrer für Aramäisch Ende der $30 \mathrm{er}$ bis in die 40er Jahre, sprach überhaupt kein Latein, und schrieb kein Deutsch. Wenn Adam und Pellikan gemeinsam den Talmud lasen, musste Adam eine Übersetzung aus dem Aramäischen ins Deutsche machen, und anschließend übersetzte Pellikan das Deutsche ins Lateinische. Es war eine ganz unangenehme Situtation für Pellikan, aber auch für Adam. ${ }^{14}$

Fähig zu sein, Christen Hebräisch zu lehren, hatte noch eine andere Dimension: Was darf man als Jude die Christen lehren? Meistens war es kein Problem, die hebräische Bibelsprache zu lehren. Wie konnten die Christen die sieben Gesetze von Noah kennen und ihnen folgen, fragte Elias Levita, wenn sie kein Hebräisch können ${ }^{15}$ Aber doch wusste man, dass auch Sprachlehre keine ganz „neutrale“ Sache war. Auch die tolerantesten christlichen Schüler hatten die Hoffnung, dass ihre jüdischen Lehrer sich taufen ließen. Clemente Abramo z. B., der spätere Lehrer von Santes Pagnini, war Hebräischübersetzer im Dienst Picos und, als er in Picos Haushalt lebte, trat er zum Christentum über. ${ }^{16}$

11 Margaritha war eine Ausnahme. Er musste aber immer wieder fürchten, dass die Universität seine Stelle an einen Minoriten übergeben wolle, da er kostenlosen Unterricht anbieten konnte. Siehe Maria Diemling: Anthonius Margaritha on the „Whole Jewish Faith". A Sixteenth-Century Convert from Judaism and his Depiction of the Jewish Religion. In: Jews, Judaism and the Reformation in Sixteenth Century German. Hg. von Dean P. Bell und Stephen G. Burnett. Leiden 2006, S. 300-33, hier 306-307.

12 Zitiert von Thomas Kaufmann: Luther and the Jews. In: Jews, Judaism and the Reformation (wie Anm. 11), S. 69-104, hier S. 85 A. 52. - Der Widmungsbrief erschien erst mit der lateinischen Übersetzung des Buches.

13 Die Amerbachkorrespondenz. Bd. 1: Die Briefe aus der Zeit Johann Amerbachs: 1481 1513. Hg. von. Alfred Hartmann. Basel 1942, S. 447.

14 Konrad Gessner berichtete, dass Pellikan usus est opera neophyti cuiusdam ex Judaeis, non satis feliciter. Christoph Zürcher: Konrad Pellikans Wirken in Zürich 1526-1556. Zürich 1975, S. 172 A. 4.

15 Jacob ben Chajim ibn Adonijah and Elias Levita. Introduction to the Rabbinic Bible and The Massoreth ha-Massoreth. Hebrew and English with explanatory notes by Christian David Ginsburg. Repr. d. 2. ed. London 1867: New York 1968, S. 99.

16 David B. Ruderman: World of a Renaissance Jew. The Life and Thought of Abraham ben Morecai Farrisol. Cincinnati 1981, S. 46. 
Noch schwieriger war die Frage, ob es für Juden erlaubt sein sollte, Christen kabbalistische Wissenschaft zu lehren. Reuchlin klagte im Widmungsbrief seiner Hebräischen Grammatik Rudimenta [1506], dass deutsche Juden entweder durch Hass oder Unkenntnis verleitet, keinen Christenmenschen in ibrer Sprache unterweisen wollen, und dies aufgrund des Einflusses eines gewissen Rabi Amos ablehnen, der im Talmud wortwörtlich sagt: ,Die Worte der heiligen Schrift werden keinen Ungläubigen erklärt .... ${ }^{17}$ R. Elia Halfan hat das Problem in einem Responsum über die Frage im Jahre 1544 diskutiert. Er verteidigte die Meinung, dass es erlaubt sei, die Bibelsprache zu lehren. Aber man dürfe keine Lehre über die mystischen oder esoterischen Geheimtraditionen erteilen. ${ }^{18}$ Aber doch ist klar, dass einige jüdische Gelehrte bereit waren, die Kabbalah zu lehren. Die Lehrer, Juden und konvertierten Juden (z. B. Yohanan Allemano und Flavius Mithridates), an Picos Hof waren nur die bekanntesten Gelehrten, die diesen Ünterrricht erteilten. R. Naftali Treves in Worms war sogar bereit, einige seiner kabbalistischen Texte auszuleihen. ${ }^{19}$

\section{Sozialer/Institutioneller Kontext der Hebraistik in Italien und Deutschland}

Es ist schon lange bekannt, dass christliche Hebraistik in Italien begann und erst später nach Deutschland gekommen ist. Ein wichtiger Grund dafür ist, dass es in Italien mehrere Plätze gab, wo "semi-neutrale“ Begegungen zwischen Christen und Juden ohne großen Skandal möglich waren und wo wissenschaftliche Informationen ausgetauscht werden konnten. ${ }^{20}$ In erster Linie hat man an die adligen und kirchlichen Höfe zu denken. Der Hof von Pico della Mirandola ist nur ein Beispiel dafür. Kardinal Dominico Grimani war auch ein Patron hebräischer Wissenschaft. Er hatte eine große Bibliothek zusammengetragen, einige Übersetzungen aus dem Hebräischen bestellt und dafür bezahlt. Grimani hatte sich auch mit Obadiah Sforno arrangiert, dass dieser Reuchlin

17 Johannes Reuchlin an Dionysius Reuchlin, Stuttgart, 7 March 1506. In: RBW. Leseausgabe. Bd. 2, S. 49 (= RBW 2, S. 45, z. 294-98, Brief Nr 138).

18 David Kaufmann: Elia Menachem Chalfan on Jews Teaching Hebrew to Non-Jews. Jewish Quarterly Review o. s. 9 (1896-97), S. 500-508. Siehe CampaninI: Reuchlins Jüdische Lehrer (wie A. 7), S. 73.

19 Eric Zrmmer: Jewish and Christian Hebraist Collaboration in Sixteenth Century Germany. Jewish Quarterly Review 71 (1980), S. 69-78, hier S. 71-72.

20 Die Phrase „semi-neutrale Begegungen“ wurde durch das "semi-neutral society" von Katz inspiriert. JАСOB KATZ: Out of the Ghetto. The Social Background of Jewish Emancipation: 1770-1870. New York 1973, S. 201. 
Hebräisch lehren werde. ${ }^{21}$ Für die Geschichte der Bibelwissenschaft sind Elias Levitas zwölf Jahre (1515-1527) als Hausgenosse des Kardinals Egidio di Viterbo sehr wichtig gewesen. Auch nach der Plünderung Roms im Jahre 1527, als Levita alle seine Güter verlor, diente Viterbo ihm noch als Patron. Mit Viterbos Unterstützung konnte Levita sein Lexikon des aramäischen Targums Meturgeman fertig schreiben. ${ }^{22}$ Wenn ein jüdischer Lehrer an einen Hof tätig war, musste er nicht seinen Umgang zu Christen begründen (obwohl andere Juden machmal solche Fragen gestellt haben!). ${ }^{23}$

Der hebräische Buchhandel, wenn man über Drucker oder Verkäufer spricht, bot auch geschäftliche Situationen, wo gelehrte Juden und Christen einander begegnen konnten. Gershom Soncino half Aldus Manutius, seine ersten Hebräischwerke zu drucken, und - nach Campanini - war er der Verfasser der kurzen Skizze der hebräischen Grammatik, die mehrmals in Manutius' Druckerei erschienen ist. ${ }^{24}$ Bomberg hatte Hebräisch von dem konvertierten Juden Felix Prato gelernt; sie haben einige Jahre als Partner in einer gemeinsamen Druckerei hebräische Bücher produziert. ${ }^{25}$ Obadjah Sforno war wahrscheinlich als Reuchlins Agent tätig, um die große Zahl hebräischer Bücher aufzuspüren, die Reuchlin kaufte. ${ }^{26}$

Eine vergleichbare Situation war fast unbekannt in Deutschland. Es gab nur ein paar Höfe, wo Interesse an Hebräisch in irgendeiner Art vorhanden war. Johann von Dalberg, Bischof von Worms, hatte ein Interesse an Hebraistik und eine eigene hebräische Bibliothek gesammelt. ${ }^{27} \mathrm{Er}$ hatte auch Reuchlin einige hebräische Handschriften für seine Bibliothek übergeben. ${ }^{28}$ Natürlich hat Reuchlin auch Hebräisch mit Jacob Jehiel Loans am kaiserlichen Hof zu Linz

21 Ariel Toaff: Sforno, Obadiah b. Jacob. Encyclopaedia Judaica. Bd. 14 (1971), S. $1209-1210$.

22 Francis X. Martin: Friar, Reformer and Renaissance Scholar. Life and Work of Giles of Viterbo 1469-1532. Villanova, Pa 1992, S. 169-171. - Bischof Georges de Selve hat Levitas Arbeit später finanziell unterstützt. WEIL, Élie Lévita (wie A. 9), S. 120-26.

23 Elias Levita fühlte sich gezwungen, eine Erklärung seiner Beziehung zu Kardinal Viterbo in "Masoreth-Ha-Masoreth" anzubieten. Vgl. JACOB BEN CHAjIm IBN AdONijah and Elias Levita. Introduction to the Rabbinic Bible (wie A. 15), S. 96-98.

24 Campanini: Reuchlins Jüdische Lehrer (wie A. 7), S. 71.

25 Shlomo Simonsohn: Some well-known Jewish Converts during the Renaissance. Revue des études juives 148 (1989) S. 17-52, hier S. 29.

26 Campanini, Reuchlins Jüdische Lehrer (wie A. 7), S. 78-79. - Es gab auch in Italien Möglichkeiten innerhalb der Klöster, Hebräisch von konvertierten Juden zu lernen. Santes Pagninus hat sein Hebräisch von dem Mönch Clemente Abramo gelernt (ebd. S. 72).

27 Johannes Reuchlin an Bischof Johannes von Dalberg, Stuttgart, nach dem 21. April 1494. In: RBW Bd. 1. S. 220, Z. 34-40; Brief Nr 64.

28 Campanini: Reuchlins Jüdische Lehrer (wie A. 7), S. 78, und Karl Preisendanz: Eine neue Handschrift aus Johann Reuchlins Bibliothek. Neue Heidelberger Jahrbücher N. F. (1936), S. 100-111, hier S. 107-108. 
gelernt. Es gab auch ein gewisses Interesse an Hebraistik unter Mönchen in deutschen Klöstern, vor allem nach der Veröffentlichung von Reuchlins Rudimenta. ${ }^{29}$ Die erste jüdische Druckerei Deutschlands wurde erst im Jahr 1530 gegründet; die ersten Druckereien waren nicht nur sehr klein, sie waren auch alle nur kurzlebig. ${ }^{30}$

Man lernt etwas über die Privatbegegungnen und den Austausch zwischen christlichen Schülern und jüdischen Helfern durch die wenigen Privatbriefe und Selbstzeugnisse. Die wichtigsten Quellen sind die Korrespondenz von Kaspar Amman, Reuchlins Korrespondenz, dazu die Hauschronik von Konrad Pellikan und die wenigen Bemerkungen in gedruckten Büchern (oder als Margainalien in den hebräischen Handschriften) von christlichen Hebräisten wie Reuchlin oder Münster. Die einzigen öffentlichen Anstalten in Deutschland, wo man ganz ohne Hemmung von jüdischer Hilfe sprechen durfte, waren die neuen Hebräischlehrstühle an vor allem protestantischen Universitäten.

Der Beitrag von Professoren jüdischer Abstammung an der Begründung von Hebraistik als Universitätsfach ist noch nicht ganz klar. Es gab z. B. fast keine Lehrer jüdischer Herkunft im frühen 16. Jahrhundert in Italien. ${ }^{31}$ Auch in Deutschland war die Zahl ganz gering. Von den 126 Hebräischlehrern, die im 16. Jahrhundert an europäischen Universitäten lehrten, waren ungefähr 30 Juden. Die 13 an deutschen Universtäts tätigen Lehrer jüdischer Abstammung sind auch nicht besonders erfolgreich gewesen. Nur sieben davon haben mehr als fünf Jahre an irgendeiner Universität gelehrt; von diesen Sieben sind nur Antonius Margaritha, Johannes Isaac und Immanuel Tremellius als Autoren bekannt geworden. ${ }^{32}$ Dieser ziemlich geringe Beitrag ist wahrscheinlich am besten dadurch zu erklären, dass ein erheblicher Teil deutscher konvertierter Juden vor ihrem Austritt (nach Elisheva Carlebachs Beschreibung) „Jüdische Funktionäre zweiten Ranges" waren, d.h. Lehrer, Kantoren, Schreiber, oder Shocheten, aber nicht gelehrte Rabbiner. ${ }^{33}$ Um den Sprung von diesen Positionen, die eine gewisse Gelehrsamkeit innerhalb der jüdischen Welt verlangten,

29 Man denkt hier vor allem an Pellikan, Sebastian Münster und Kaspar Amman, aber auch an ihre weniger bekannten Zeitgenossen wie die Mönche von Ottobeuren, die gute Kontakte zu Reuchlin hatten.

30 Stephen G. Burnett: German Jewish Printing in the Reformation Era (1530-1633). In: Jews, Judaism and the Reformation (wie Anm. 11), S. 503-527, hier S. 506-510.

31 Paul Grendler: The Universities of the Italian Renaissance. Baltimore \& London 2002, S. 102/3, S. 230/1. - Rom war eine Ausnahme, weil Hebräisch seit der Zeit von Leo X. ständig gelehrt wurde. Der Lehrstuhl für Hebräisch am Collegium Romanum wurde im Jahre 1553 gegründet und war dann fast ständig besetzt. Vgl. RicCArdo G. Villoslada: Storia del Collegio Romano dal suo inizio (1551) alla soppressione della Compagnia di Gesu (1773). Rom 1954, S. 326.

32 Vgl. Anhang.

33 Elisheva Carlebach: Divided Souls: Converts from Judaism in Germany, 15001750. New Haven 2001, S. 125-26. 
auf einen Lehrstuhl zu schaffen, bedurfte es vieler Anstrengungen. Margaritha hat es an der Universität Wien geschafft, aber immer wieder musste er fürchten, dass seine Stelle einem Bettelmönch (der kostenlos lehren würde) übergeben würde. ${ }^{34}$ Johannes Isaac hat eine Weile gebraucht, bis er seinen Lehrstuhl im richtigen Stil wahrnehmen konnte, d.h. er musste Latein ganz gründlich an der Universität Löwen lernen und dazu Sprachpädagogik, die von christlichen Lehrern verwendet wurde. Jahre später klagte Andreas van Gennep Balenus, Ordinarius für Hebräisch zu Löwen, dass Johannes Isaac Balenus' grammatikalische Arbeiten unter seinem eigenen Namen veröffentlicht hatte. ${ }^{35}$ Nur Immanuel Tremellius hatte einen guten Ruf von Anfang an, aber er hatte schon als junger Mann eine Ausbildung an der Universität Padua erhalten und nach seiner Konversion zum Protestantismus war er bereit, einen prominenten Platz an einer Universität zu bekleiden. ${ }^{36}$

\section{Dienstleistungen jüdischer Vermittler der hebräischen Sprache}

Wenn man über jüdische Hilfe beim Hebräischunterricht für Christen am Ende des Mittelalters spricht, ist der Unterricht selbst am wichtigsten. Aber es reichte für christliche Schüler nicht aus, dass sie Buchstaben und Grammatik lernten. Sie mussten auch Zugang zu jüdischen Texten haben. Das meint das Abschreiben von handschriftlichen Texten, die Vermittlung des Verkaufs von Handschriften oder gedruckten Büchern oder sogar die Übersetzung von Texten. Nicht alle christlichen Hebräischschüler haben alle diese Dienste gebraucht oder gewünscht. Es ist gewiss auch wahr, dass nicht alle jüdischen Helfer alle diese Rollen bekleidet haben. Aber wenn man von dem ganzen Phänomen jüdischer Vermittlung des Hebräischen spricht, müssen alle diese Aktivitäten betrachtet werden.

Der Hebräischunterricht war die wichtigste Leistung, die jüdische Vermittler des Hebräischen ihren christlichen Gesprächspartnern anbieten konnten. Aber es war keine einfaches Verfahren. Wenn man vom Hebräischunterricht des Spätmittelalters sprechen will, muss man einen Unterschied zwischen askenasischer und sephardischer Praxis machen. Juden sowohl im nördlichen wie im südlichen Europa haben mit dem Unterricht schon in der Familie angefangen,

34 Diemling: Anthonius Margaritha (wie A. 11), S. 306/7.

35 Henry de Vоснт: History of the Foundation and the Rise of the Collegium Trilingue Lovaniense 1517-1550, 4 Bde. Nendeln, Liechtenstein 1980, Bd. 4, S. 300/1.

36 Kenneth Austin: Immanuel Tremellius (1510-1580), the Jews and Christian Hebraica. In: Bundeseinheit und Gottesvolk. Reformierter Protestantismus und Judentum im Europa des 16. und 17. Jahrhunderts. Hg. von Achim Detmers und Jan Marius J. Lange van Ravenswaay. (Emder Beiträge zum reformierten Protestantismus, Bd. 9) Wuppertal 2005, S. $71-88$. 
durch Zuhören während des Gottesdienstes, das Lernen von Buchstaben, Vokabeln, und manchmal durch Lautlesen. Mit dem Anfang von formellem Unterricht wurde erwartet, dass Schüler zunächst die Buchstaben und Vokalzeichen lernten, damit man Worte und nachher Gebete und Bibelverse laut lesen konnte, um am Gottesdienst teilzunehmen. ${ }^{37}$ Wenn man die Aussprache der Wörter lernt, bekommt man zugleich eine Übersetzung der einzelnen Wörter. Nachdem der Lehrer ein Wort aus einem Vers übersetzt, wiederholt der Schüler die Übersetzung, bis alle Schüler ein Wort im Vers wiederholt haben. ${ }^{38}$ Die Schüler lernten Hebräisch zu lesen, wenn sie diese wöchentlichen Abschnitte übten und dann in die Landessprache übersetzten. In der aschkenasischen Welt wurde auch das Lesen von Targum Onkelos und des Pentateuchkommentars Raschis ein Bestandteil des Unterrichts. ${ }^{39}$ Im sephardischen Bereich aber wurde es nach dem muslimischen pädagogischen Vorbild üblich, grammatikalischen Unterricht mit dem Bibelunterricht zu kombinieren. Es war ein Ziel des Unterrichts, nicht nur das Lesen zu lehren, sondern auch eine bessere Kenntnis der Grammatik zu vermitteln. ${ }^{40}$ Deshalb haben jüdische Autoren wie die Familie Kimhi auch Grammatikbücher verfasst, um diesen Unterricht zu unterstützen. Diese Grammatikbücher waren wichtige Hilfsmittel auch für christliche Hebräischschüler, vor allem, nachdem sie ins Lateinische übersetzt worden waren.

Die aschkenasische pädagogische Praxis war nicht geeignet für christliche Schüler, weil sie fast keine Verbindung mit der gesprochenen hebräischen Sprache hatten. Mit einem Tutor konnte man natürlich etwas Hebräisch oder

37 Jacob Katz: Tradition and Crisis. Jewish Society at the End of the Middle Ages. Übers. von Bernard Dov Cooperman. New York 1993, S. 156-58, S. 162/3.

38 Isidore Fishman: The History of Jewish Education in Central Europe From the End of the Sixteenth Century to the End of the Eighteenth Century. London 1944, S. 95. Nach Fishman (ebd. S. 90) wurde das Gebetbuch als Lehrbuch verwendet. „There were no elementary primers from which reading was practiced, the Prayer Book being used for the purpose. Individual letters with various sounds were pointed out in the Prayer Book, and until they were thoroughly known the teacher was advised not to begin instruction in the formation of words. ... By this means the pupil became familiar with the Hebrew and learned to read it correctly. He was also taught incidentally the translation of individual words and the inflection of nouns." Fishmans Bemerkungen stammen vom Abraham b. Moddel of Öttingen: „Maarechet Abraham“ [„Die Ordnung Abrahams“] (Fürth 1769). "Seyfer beeyr Moushe“ (Prag 1604) bietet ein Beispiel dieser Art Sprachunterricht des frühen 17. Jahrhunderts. - Jean Baumgarten: Introduction to Old Yiddish Literature. Hg. und übers. von Jerold C. Frakes. Oxford 2005, S. $102-$ 103.

39 „Huqqei ha-Torah“, [= „Gesetze der Lehre,“ geschrieben ca. 1309], übers. von Moritz GüDEMANN: Geschichte des Erziehungswesens und der Cultur der abendländischen Juden während des Mittelalters und der neueren Zeit. 3 Bde. Amsterdam 1966, Bd. 1, S. 104/5. - Siehe auch Ephraim Kanafogel: Jewish Education and Society in the High Middle Ages. Detroit 1992, S. 30/1.

40 Ivan G. Marcus: Schools, Jewish. In: Dictionary of the Middle Ages. Bd. 11, S. 70-72. 
Aramäisch lernen. Man denkt hier an die Talmudstunden von Michael Adam und Pellikan. Aber um wirklich erfolgreich zu sein, mussten jüdische Lehrer die christliche Pädagogik, das Lernen von grammatikalischen Formen nach dem Vorbild des Griechischen und Lateinischen lernen. Antonius Margaritha behauptete ganz prahlerisch, dass ein Christ, der bei ihm Hebräisch ein Jahr lerne, mehr Grammatik als die meisten Juden kenne. ${ }^{41}$

Das Abschreiben von Handschriften war wichtig vor und auch nach dem Wachstum des hebräischen Buchmarktes. Reuchlin und Pellikan haben beide mit Böhmes Handschrift von Moses Kimhis Grammatik gearbeitet und Pellikan hat sogar die Fragmente selbst abgeschrieben. Andere reichere Christen haben jüdische Schreiber bezahlt, Handschriften zu kopien. Das Kopieren war besonders wichtig für Handschriften kabbalistischer Bücher. Paulus Aemilius war bei Johann Widmanstetter als Schreiber tätig und er trat als Bediensteter von Widmanstetter zum Christentum über. ${ }^{42}$ Levita hat Handschriften für Viterbo kopiert. ${ }^{43}$ Die Bereitschaft zum Ausleihen von Handschriften, nach dem Beispiel von R. Naftali Treves in Worms, war nicht so üblich wie das Abschreiben oder der Verkauf von Handschriften, aber es war auch keine Ausnahme. ${ }^{44}$

Juden haben auch als Vermittler oder Käufer von Büchern und Handschriften gedient. Im Jahre 1498 hat Reuchlin während seines Aufenthalts in Rom eine große Zahl hebräischer Bücher innerhalb von ein paar Monaten gekauft. Campanini hat diese Tatsache als Beweis für die Hilfe Obadiah Sfornos außerhalb des Unterrichts vorgelegt. Durch die Vermittlung von Jacob Jehiel Loans hat Reuchlin eine große Bibelhandschrift (das Codex Reuchlin 1) vom Kaiser bekommen. ${ }^{45}$ Für die Beschaffung besonderer Bücher und Handschriften bleibt jüdische Hilfe während des gesamten 16. Jahrhundert eine Notwendigkeit, aber einige jüdische Bücher sind zugänglicher geworden. Die eindrucksvollen Sammlungen von hebräischen Handschriften von Pico della Mirandola (123 hebräische Hss.) und Kardinal Grimani (193 hebräische Hss. ${ }^{46}$ sowie die

41 Diemling: Anthonius Margaritha (wie A. 11), S. 323, A. 69.

42 Hans Striedl: Paulus Aemilius an J. A. Widmanstetter. Briefe von 1543/44 und 1549. Aus dem Hebräischen übersetzt und kommentiert. In: Ars Iocundissima. Festschrift für Kurt Dorfmüller zum 60. Geburtstag. Hg. von Horst Leuchtmann und Robert MüNSTER. Tutzing 1984, S. 333-56, hier S. 333.

43 Martin, Friar, Reformer and Renaissance Scholar (wie A. 22), S. 166.

44 Die Vorlage für den Cod. Heb. 285 der Bayerische SB stammt z. T. aus eine Hs., die Jacob Martino gehörte. Widmanstetter schrieb: Ex codice Zoharis quem habet Jac. Martino Hebr. Romae A. 1537 Franc. Parnassus scribit. Zitiert von Moritz SteinSCHNEIDER, Die hebraeischen Handschriften der K. Hof- und Staatsbibliothek in Muenchen. Zweite Aufl. München 1895, S. 147.

45 Campanini: Reuchlins Jüdische Lehrer (wie A. 7), S. 77-79.

46 Über die Bibliotheken von Pico (später von Grimani gekauft) und Grimani selbst, s. Giuliano Tamani: I Libri Ebraici del Cardinal Domenico Grimani. Annali di 
von Johann Albrecht von Widmanstetter (136 hebräische Hss.) wurden durch jüdische Hilfe per Abschreiben und Verkauf von Handschriften möglich gemacht. $^{47}$

Nach 1500 wurde es leichter für Christen, selber hebräische Bücher zu kaufen. Schon im Jahre 1500 hatte Pellikan seine erste hebräische Bibel (eine Soncino Ausgabe) bei einem Buchverkäufer in Tübingen gefunden. ${ }^{48}$ Reuchlin konnte im Jahre 1506 mit großer Zuversicht schreiben, dass überall in Italien hebräische Bibeln gedruckt werden, die sich ein jeder für weniger Geld mühelos erwerben kann .... ${ }^{49}$ Etwas später hat Daniel Bomberg seine Bücher südlich und nördlich der Alpen verkauft. Melanchthon, z. B., kaufte durch einen Agenten ein Exemplar der Biblia rabbinica [1517] auf der Leipziger Messe im Jahre $1518 . .^{50}$

Die Übersetzung jüdischer Texte spielt auch eine Rolle in der Vermittlung der hebräischen Sprache. An erster Stelle denkt man an Picos Übersetzer Yohanan Allemano Elijah del Medigo und Flavius Mithridates und ihre ganz tüchtige Arbeit mit hebräischen (vor allem kabbalistischen) Texten. ${ }^{51}$ Paulus Ricius hat Josef Gikatillas Portae lucis [Tore des Lichts] in einer verkürzten lateinischen Übersetzung im Jahre 1516 veröffentlicht. ${ }^{52}$ Felix Prato hat kabba-

Ca'Foscari. Rivista della Facolta di lingue e letterature straniere dell'Universita di Venezia 34/3 (1995), S. 5-52, hier S. 7 (Zahl von Picos Hss.), S. 14-42 (Grimani).

47 Über Widmanstetters Sammlung, s. Steinschneider: Die hebraeischen Handschriften (wie Anm. 44), S. 266 [Register] und Hans Striedl: Die Bücherei des Orientalisten Johann Albrecht Widmanstetter. In: Serta Monacensia. Franz Babingen zum 15. Januar 1951 als Festgruß dargebracht. Hg. von Hans Joachim Kissling und Alois Schmaus, Leiden 1952, S. 200-244, hier S. 218-19 (inkl. Korrektur von STEInschneiders Register zu Widmanstetters Hss.).

48 Pellican: Hauschronik (wie A. 1), S. 22.

49 Reuchlin an Dionysius Reuchlin, Stuttgart, 7. März 1506. In: RBW. Leseausgabe. Bd. 2, S. 36 (= RBW 2, S. 35, Z. 29-30; Brief Nr. 138). 1491 schrieb Johannes Strehler, Reuchlins Agent in Florenz, dass er keine hebräische Bibel gefunden habe, ausser der einen aus Bologna, der jedoch etliche Blätter fehlen und die ich deshalb nicht kaufen will. Johannes Strehler an Reuchlin, Florenz, 25. November 1491. In: RBW. Leseausgabe. Bd. 1, S. 106 (= RBW 1, S. 149, Z. 7-8; Brief Nr. 48).

50 Philipp Melanchthon an [Georg Spalatin - Wittenberg, 24 September 1518], Melanchthons Briefwechsel. Kritische und kommentierte Gesamtausgabe. Hg. von Heinz Scheible, Stuttgart-Bad Cannstatt 1991 ff, Bd. 1, S. 75, Z. 1-3 (Brief Nr. 24).

51 Joseph Dan: The Christian Kabbalah: Jewish Mystical Books and Their Christian Interpreters. Cambridge 1997, S. 201-203. - Zu Del Medigos Übersetzungen von hebräischen philosophischen Büchern s. M. David GeFFen: Faith and Reason in Elijah Del Medigo's „Behinat Ha-Daat“ and the Philosophic Backgrounds of the Work. Ph.D. diss. 1970; Nachdr.: Ann Arbor, Mich. 1971, S. 11-20, S. 25-26.

52 Ebd., 197. - Siehe Bernd Roling: Prinzip, Intellekt und Allegorese im Werk des Christlichen Kabbalisten Paolo Ricci (gest. 1541). In: An der Schwelle zur Moderne. Juden in der Renaissance. Hg. von Giuseppe Veltri und Annette Winkelman. Leiden 2003, S. $155-187$. 
listische Bücher für Kardinal Viterbo übersetzt. ${ }^{53}$ Aber nichtkabbalistische Werke wurden auch übersetzt. Obadjah Sforno und Abraham de Balmes haben beide zweisprachige Grammatiken verfasst; de Balmes' Grammatik wurde nach seinem Tod bei Bomberg veröffentlicht. ${ }^{54}$

In Verbindung mit diesen Leistungen - Lehren, Abschreiben, Kaufen, Übersetzen - wurde das Wort „Dienstleistung“ betont, und das ist kein Zufall. Dieser Austausch von Informationen geht meistens von Juden im Richtung Christen. Es gab natürlich gewisse Ausnahmen. Elias Levita hat Griechisch bei Kardinal Viterbo gelernt und war deshalb nicht nur ein Klient. Yohanan Allemano hat sicherlich auch von Pico gelernt, obwohl er älter als Pico war und seine Vorstellungen über Kabbalah und Philosophie waren gründlicher durchdacht. ${ }^{55}$

Aber meistens wurde Hebraistik als Handel, Dienstleistung gegen Zahlung verstanden. Viele Hebräisten klagten über die Kosten des Handels. Für Wolfgang Capito war es ganz klar, dass Matthaeus Adrianus zu viel Geld forderte und zu wenig Unterricht bot. ${ }^{56}$ Immerhin muss man sagen, dass die Mehrheit der mit jüdischen Bibelkommentaren arbeitenden deutschen Hebräisten (Capito, Münster, Oecolampadius, Reuchlin) Schüler von Adrianus waren. ${ }^{57}$ Jan van Campen, Professor des Hebräischen zu Löwen, musste Elias Levita zwei Dukaten pro Monat für den Unterricht zahlen. ${ }^{58}$ Melanchthon berichtete, dass Reuchlin einen Dukaten pro Unterrichtsstunde an Sforno zahlen musste, eine Schätzung, die wahrscheinlich etwas übertrieben war. ${ }^{59}$ In diesem Falle haben sicherlich die jüdischen Teilnehmer des Austausches ihr Geld bekommen und fast sicher auch verdient.

Aber solche "Geschäfte" haben nicht immer geklappt, vor allem für die Schüler. Man hört nicht selten von Inkompetenz jüdischer Lehrer bzw. Helfer oder sogar von Betrug. Pellikan klagte mehrmals, dass er immer wieder auf seinen Reisen jüdische Lehrer suchte und als er endlich in Frankfurt/Main zwei

53 Simonsohn. Some well-known Jewish Converts (wie A. 25). S. 28.

54 Campanini: Reuchlins Jüdische Lehrer (wie A. 7), S. 82, und Anthony J. Klijnsmit: Standstill or Innovation? Helmantica 44 (1998), S. 39-71, hier S. 49-50.

55 Moshe Idel: The Magical and Neoplatonic Interpretations of the Kabbalah in the Renaissance. In: Essential Papers on Jewish Culture in Renaissance and Baroque Italy. Hg. von David B. Ruderman, New York 1992, S. 111.

56 WILlI: Beitrag des Hebräischen (wie A. 2), S. 144 und A 43.

57 Stephen G. Burnett: Reassessing the „Basel-Wittenberg Conflict: Dimensions of the Reformation-Era. Discussion of Hebrew Scholarship. In: Hebraica Veritas? Christian Hebraists and the Study of Judaism in Early Modern Europe. Hg. von Allison P. Coudert und Jeffrey S. Shoulsen. Philadelphia 2004, S. 181-201, hier S. 183-84.

58 Jan van Campen an Dantiscus, Venedig, 4. Februar 1535. Zitiert von DE Vocht: History of the Foundation (wie A. 35), Bd. 3, S. 201, A. 1.

59 Campanini: Reuchlins Jüdische Lehrer (wie A. 7), S. 77-78. 
hilfsbereite Rabbiner fand, waren sie unfähig, seine Fragen zu beantworten. ${ }^{60}$ Ebenso hat der Priester Johannes Böhm in Ulm einem Juden viel Geld bezahlt, damit er eine deutsche Übersetzung von den zwei grammatikalischen Fragmenten von Moses Kimhis Grammatik machte. Nach Pellican hatte der Jude überhaupt keine Verständnis von Grammatik gehabt und seine Übersetzung war ganz wertlos. ${ }^{61}$ Johannes Forster, einer von Reuchlins letzten Studenten, schimpfte: Wenn es einen gibt, der seine Fähigkeiten an den Rabbinen verschwendet, der sie in seinem Hause auf eigene Kosten als Lehrer unterhalten, der sich oft und lange in ihren Synagogen herumgetrieben und ihre Commentare fleißig gelesen hat, dann ist es Forster, und dennoch habe ich nichts Ausgezeichnetes, nichts was besonderen Lobes werth wäre davongetragen. ${ }^{62}$ Am Ende seiner Karriere hat Forster (vergeblich) versucht, die Hebraistik ganz neu zu gestalten, um sie ganz unabhängig von ihrer jüdischen Vergangenheit zu machen.

\section{Schlussbemerkungen}

Die Brücke zwischen den wissenschaftlichen Sphären der Juden und Christen, die von den jüdischen Vermittlern der Hebraistik bis zum Ende des Mittlealters gebaut worden war, war sehr schmal. Die Eigenschaften, Fähigkeiten und beruflichen Tätigkeiten der geringen Zahl der Hebräischlehrer, Buchkäufer, Schreiber, und Übersetzer - Vermittler jeder Art - haben manchmal einen direkten Einfluss auf die Karriere und Leistungen ihrer christlichen Schüler gehabt. Da die Beziehung zwischen Lehrern und Schüler in zwei Richtungen geht, die manchmal verfehlten Versuche, Hebräisch von ihren jüdischen Lehrern zu lernen, haben auch einen Einfluss auf die Entwicklung christlicher Hebraistik gehabt. Man denkt hier vor allem an den eigenartigen Versuch des Lutheraners Johannes Forster, Hebräisch ganz ohne Referenz auf jüdische Lehrer oder Autoren zu lehren. Mit dem Wachstum eines christlichen hebräischen Buchmarkts sind mehrere Bücher auf hebräisch, die bestimmt für christliche Kunden ge-

60 Pellican: Hauschronik (wie A. 1), S. 50.

61 Ebd. 21.

62 Si quis est, qui facultates suas in Rabinos profudit, qui in ipsorum synagogis longo tempore versatus est, qui domi propriis impensis ipsos preceptores aluit, qui ipsorum commentaria studiose evoluit, Forsterus est, \& tamen ... nihil eximii, \& quod singulari laude dignum esset, reportavi. "Johannes Forster: Dictionarium Hebraicum Novum, Non ex Rabinorum Commentis, nec nostratium doctorum stulta imitatione descriptum, sed ex ipsis thesauris sacrorum Bibliorum \& eorundem accurata locorum collatione depromtum, cum phrasibus scripturae Veteris \& Novi Testamenti diligenter annotatis." Basel 1557, S. 5". (Basel UB). Uebers. von Ludwig Geiger: Das Studium der Hebräischen Sprache in Deutschland vom Ende des XV. bis zur Mitte des XVI. Jahrhunderts. Breslau 1870, S. 100. 
schrieben und gedruckt waren, erhältlich. Daher war jüdische Hilfe beim Lernen des Hebräischen unter Anfängern im späteren 16. Jahrhundert nicht mehr so nötig. ${ }^{63}$ Für Studenten, die den Talmud oder die Kabbalah lesen wollten, blieb die Hilfe jüdischer Lehrer fast unentbehrlich, auch im 17. Jahrhundert. ${ }^{64}$

Die Produktion und Vermittlung von jüdischen Texten war auch ein Teil des Austauschs zwischen Juden und Christen. Auch das leichteste Verfahren, das Kaufen von hebräischen Büchern, brauchte manchmal jüdische Hilfe, um die richtigen Titel zu finden. Im Jahre 1546 war Konrad Pellikan wütend, als sein Schwager Johannes Fries viele wertlose jüdische Bücher in Italien gekauft hatte. Nach Pellikans Meinung war unter den Büchern sehr vieles ganz nutz und werthlos [...]. Fries hatte sich eben in seiner Unkenntnis durch jüdische Arglist täuschen lassen und ohne alle Prüfung eingekauft. ${ }^{65}$ Ein jüdischer Agent, der Hebräisch lesen konnte, hätte es wahrscheinlich besser machen können.

Christliche Hebräischschüler haben nicht selten am Ende des Mittelalters ihre eigenen Abschriften von hebräischen Handschriften gemacht. Konrad Pellikan hat mehrmals eigene Kopien gemacht, weil er sich wahrscheinlich die Hilfe eines Schreibers während seiner Zeit als Fransziskanermönch nicht leisten konnte. Sein Student Sebastian Münster hat auch Reuchlins Exemplar des Sefer Nizzahons abgeschrieben. ${ }^{66}$ Aber die Christen, die einen Schreiber anstellen konnten, haben nicht selten sehr wertvolle Bücher bewahrt. Pico della Mirandola, Egidio di Viterbo und Johann Albrecht von Widmanstetter haben sich um die Hebraistik und Judaistik sehr verdient gemacht, indem sie Schreiber wie Elias Levita und andere angestellt haben, um weitere Kopien von seltenen handschriftlichen Büchern zu machen.

Jüdische Übersetzer haben das Verdienst, nicht nur für Schüler der Hebraistik, sondern auch für andere christliche Leser, die überhaupt keine Hebräisch kannten, Texte zugänglich gemacht zu haben. Mit Hilfe von Büchern wie z. B. Paulus Ricius Porta Lucis bekommt man einen ersten Einblick in einen der wichtigsten Texte der Kabbala.

Die kulturelle Wirkung dieser jüdischen Vermittler des Hebräischen war ganz enorm, vor allem wenn man ihre geringe Zahl beachtet. Aber so ist es oft in der jüdischen Geschichte: Ein geringe Zahl von Leuten übt einen großen

63 Stephen G. Burnett: Christian Hebrew Printing in the Sixteenth Century. Printers, humanism, and the Impact of the Reformation. Helmantica 51/154 (2000), S. 13-42.

64 Peter T. van Rooden: Theology, Biblical Scholarship and Rabbinical Studies in the Seventeenth Century. Constantijn L'Empereur (1591-1648) Professor of Hebrew and Theology at Leiden. Leiden 1989, S. 163-66, 163, Anm. 270-71.

65 Pellikan: Hauschronik (wie A. 1), S. 162.

66 Stephen G. Burnett: A Dialogue of the Deaf. Hebrew Pedagogy and Anti-Jewish Polemics in Sebastian Münster's Messiahs of the Christians and the Jews (1529/30). Archiv für Reformationsgeschichte 91 (2000), S. 168-190, hier S. 176. 
Einfluss auf die Mehrheitsgesellschaft aus, der viel erheblicher ist, als man den blossen Zahlen nach schätzen oder erwarten könnte.

\title{
ANHANG
}

\author{
Jüdische Hebräischlehrer
}

\section{Universitätsstudium}

\section{Italien}

Abraham de Balmes:

Obadiah Sforno:

Jacob Martino

*Flavius Mithridates

\section{Deutschland}

*Adrianus, Matthaeus

*Aemilius, Paul

*Ricius, Paulus

*Tremellius, Immanuel

*Weidner, Paul

\section{Ohne Universitätsverbindung}

\section{Italien}

Carlo Calonimos

*Clemente Abramo

Elia del Medigo

Elia Menahem Halfan

Elias Levita

*Felix Prato

Jacob Gabbai

Gershom Soncino

*Konvertierte Juden
Dr. Med. Naples 1492

Dr. Med. Ferrara, 1501

Medizinstudium Padua, 1503-06

Theologiestudium Napoli, c. 1474-77

Medizinstudium in Italien

Medizinstudium in Tübingen

Studium in Padua, D Med Pavia vor 1506

Studium in Padua, ca. $1530-40$

Medizinstudium Padua

\section{Deutschland}

Jacob b. Yehiel Loans (von Mantua)

Kalman

R. Liva [Loeb] (Worms)

R. Moshe Moelln

R. Naftali Treves (Worms)

III. Jüdische Universitätslehrer des Hebräischen im 16. Jh. in Deutschland

Adrianus, Matthaeus - 4 Jahre

Aemilius, Paul - 28 Jahre

Antonius Jud, Strasbourg - 4 Jahre

Berhard Göppingen - 2 Jahre

Cellarius, Johannes - 4 Jahre 
David Leonhard - 6 Jahre

Isaac, Johannes - 13 Jahre

Isaac, Stephen - 20 Jahre

Margaritha, Antonius - 12 Jahre

Staffelsteiner, Paul - 4 Jahre

Storch, Jacob - 5 Jahre

Tremellius, Immanuel - 26 Jahre

Weidner, Paul - 24 Jahre 